\title{
SiCloud: an online education tool for silicon photonics
}

Cathy Jiang, Peter T. DeVore, Cejo Konuparamban Lonappan, Jost Adam, Bahram Jalali

Cathy Y. Jiang, Peter T. S. DeVore, Cejo Konuparamban Lonappan, Jost Adam, Bahram Jalali, "SiCloud: an online education tool for silicon photonics," Proc. SPIE 10452, 14th Conference on Education and Training in Optics and Photonics: ETOP 2017, 104521Q (16 August 2017); doi: 10.1117/12.2266424

SDIE Event: 14th Conference on Education and Training in Optics and Photonics, ETOP 2017, 2017, Hangzhou, China 


\title{
SiCloud: An Online Education Tool for Silicon Photonics
}

\author{
Cathy Y. Jiang ${ }^{\mathrm{a}, * 1^{*}}$, Peter T.S. DeVore ${ }^{\mathrm{a}}$, Cejo Konuparamban Lonappan ${ }^{\mathrm{a}}$, Jost Adam ${ }^{\mathrm{b}}$, Bahram \\ Jalali $^{\mathrm{a}}$ \\ ${ }^{a}$ Dept. of Electrical Engineering, University of California, Los Angeles, CA USA 90024 \\ ${ }^{\mathrm{b}}$ Mads Clausen Institute, University of Southern Denmark, DK-6400 Sønderborg, Denmark
}

\begin{abstract}
The silicon photonics industry is projected to be a multibillion dollar industry driven by the growth of data centers. In this work, we present an interactive online tool for silicon photonics. Silicon Photonics Cloud (SiCloud.org) is an easy to use instructional tool for optical properties of silicon and related materials, waveguide design and modal simulations as well as information capacity of silicon channels.
\end{abstract}

Keywords: Silicon Photonics; Optical Interconnects; Engineering education; Silicon-On-Insulator; Finite Element Method; nanowire waveguide; Propagating Modes; Information Capacity

\section{INTRODUCTION}

The silicon photonics industry is projected to grow rapidly owing to the increasing demand for higher data bandwidth for telecom and datacenter applications [1,2]. Becoming an expert in this field is challenging for researchers and students alike, as design of silicon photonic circuits and systems requires understanding of diverse areas such as material science, semiconductor physics, electronics, and waveguide design. Effective educational design tools that bridge these areas for developing integrated silicon photonics solutions are therefore needed to train the next generation of engineers who will fuel this burgeoning industry. The SiCloud initiative (Silicon Photonics Cloud - SiCloud.org) is an attempt to address this need. The homepage of SiCloud.org is shown in Figure 1.
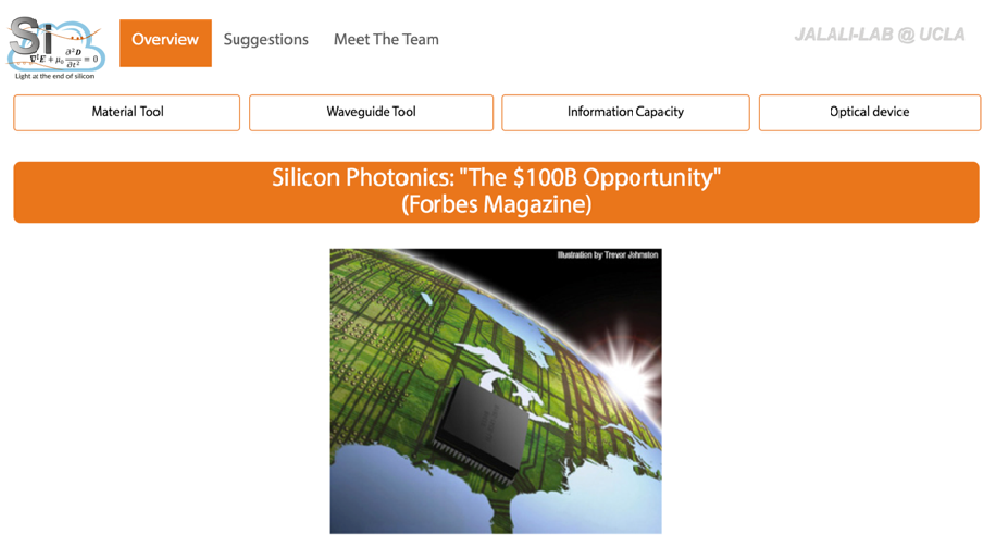

Figure 1. Homepage of Silicon Photonics Cloud, the first web-based interactive tool for silicon photonics

SiCloud.org is a website designed to be a one-stop silicon photonics resource for students, researchers, and design engineers. SiCloud version 1.0 debuted in June of 2014, and includes material parameters and their wavelength dependence for silicon and other related materials $[3,4]$. A user-friendly interface on the website provides the users with an interactive visualization of both linear and nonlinear properties of important silicon photonics materials using data from classic handbooks to the latest publications.

1*yunshanjiang@gmail.com

14th Conference on Education and Training in Optics and Photonics: ETOP 2017, edited by Xu Liu,

Xi-Cheng Zhang, Proc. of SPIE Vol. 10452, 104521Q · ( 2017 ICO, IEEE, OSA, SPIE

CCC code: $0277-786 X / 17 / \$ 18 \cdot$ doi: $10.1117 / 12.2266424$

Proc. of SPIE Vol. 10452 104521Q-1 
We report the next version of SiCloud featuring interactive tools for analyzing and visualizing propagation mode parameters for a wide range of wavelengths and submicron geometries of waveguides, as well as the spatial electromagnetic field distributions. SiCloud's expanded capability makes it a reference tool for validating silicon photonics design simulations and experimental results. The latest addition of features to SiCloud also includes a unique tool for estimating the information capacity of custom designed silicon waveguides as a function of signal intensity. This feature allows the user to explore the limits to information capacity imposed by the nonlinear optical interactions in silicon nanowires.

\section{Online Tools}

\subsection{Material Tool}

The user interface of the material tool is shown in Figure 2. The material tool enables interactive visualization of the optical properties of the most commonly used materials in silicon photonics. In addition to silicon and silicon nitride, the tool also covers other materials such as copper, tungsten, and aluminum used for metallization; silicon oxide, and sapphire used as insulators in silicon-on-insulator (SOI) substrate; and germanium used as optical detector. Refractive index, and extinction coefficient data of these materials from classic optical handbooks [5], and the well-known Sellmeier fitting equations are illustrated for the user provided wavelength range. Silicon waveguides with high index contrast enables nanoscale confinement which in turn enhances the nonlinear effect. The nonlinear properties of silicon have been exploited for many applications [6,7]. Therefore, we also included Kerr, Raman, and two-photon absorption coefficients in the visualizations, based on recently reported measurements [8].

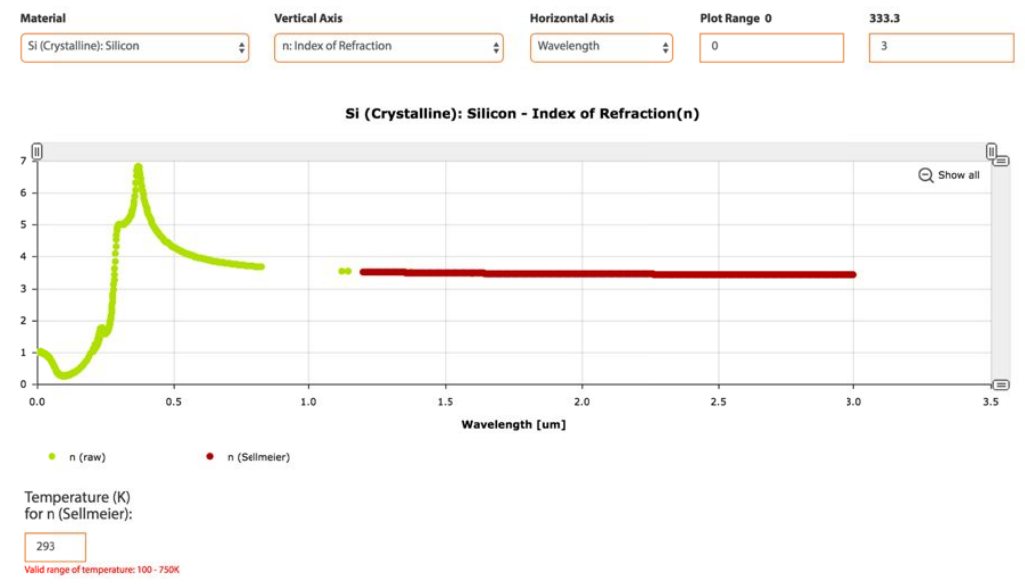

Figure 2. The material tool provides interactive visualizations of linear and nonlinear parameters of silicon and other related materials. The index of refraction of silicon from visible to mid-infrared are shown, including the measured data (green) from handbooks and calculated data (red) from Sellmeier fitting equation. 


\subsection{Waveguide Tool}

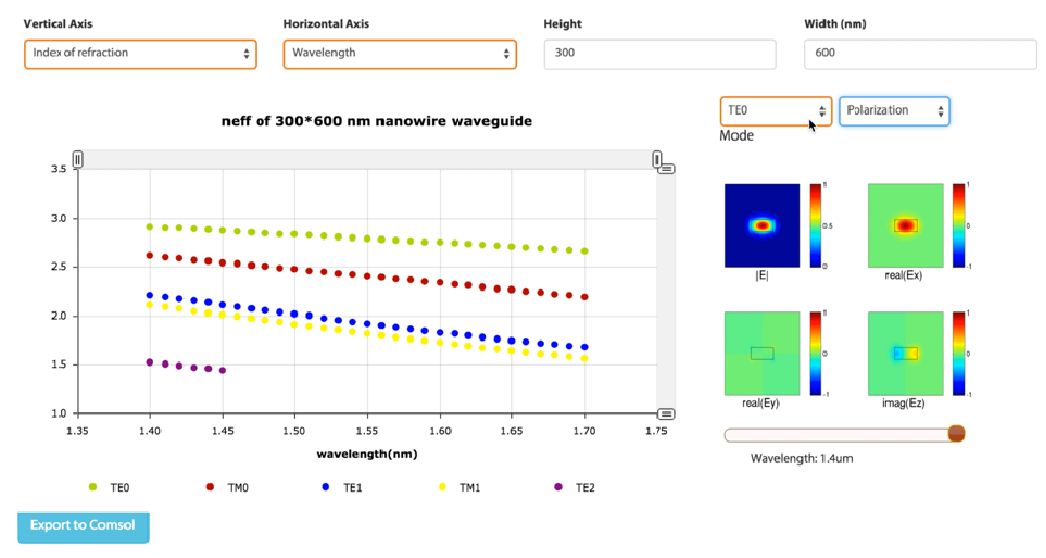

Figure 3. Waveguide tool illustrates the change of propagation parameters of guided modes in slot waveguides, as a function of height, width, or wavelength. The mode gallery on the right interactively plots the mode intensity and the electric filed distributions in three dimensions. TE0 mode of 300x600 waveguide at 1.4um is shown above. The data pre-calculated using wave optics module of COMSOL, and the source files are provided for downloading.

Submicron SOI slot waveguides space-efficient and enable strong confinement for the propagating mode [9]. The propagation constant and mode distribution are two of the key features that characterizing a guided. They vary as functions of the mode dimensions (height and width) and wavelength. The waveguide tool allows visualization of effective modal parameters as well as the spatial electromagnetic field distributions across a wide range of relevant telecommunication wavelengths and mode geometries. The parameters include refractive index, group velocity, group velocity dispersion, higher order dispersion, and Kerr nonlinear coefficients (Figure 3,4). The data was pre-calculated using Finite-Element-Method (FEM) simulations employing the wave optics module of COMSOL [10]. The COMOSL source files are available for download for custom simulations.

The waveguide tool can not only be used for estimating mode dispersion, nonlinear effects, and mode coupling strength, but also serve as a reference to compare simulation and experimental results.

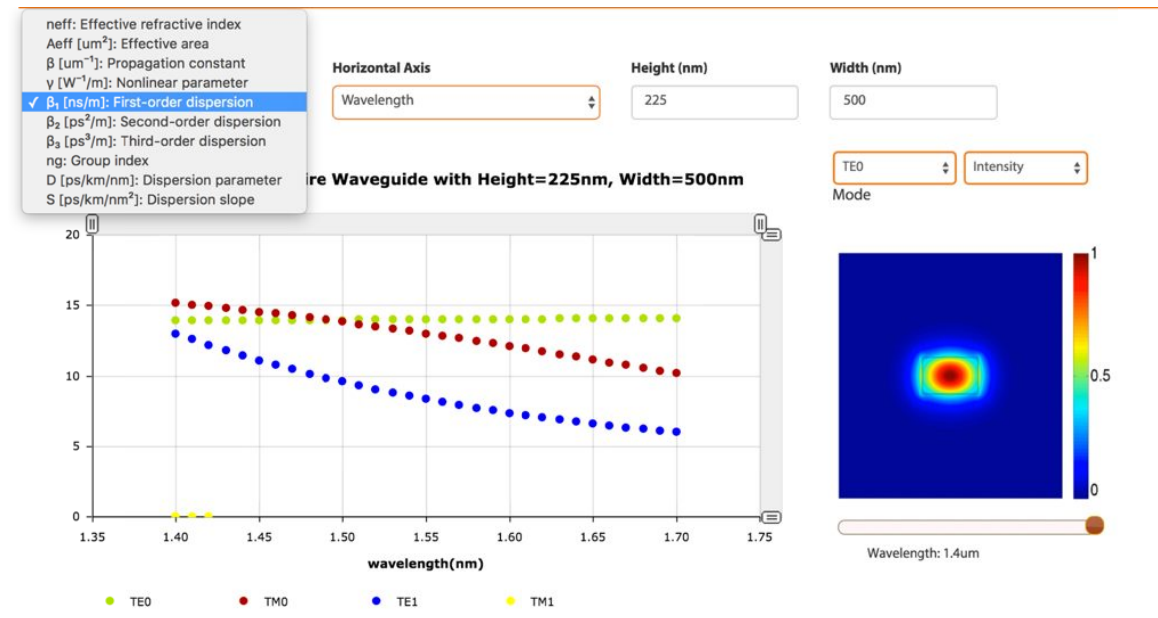

Figure 4. Group velocity dispersion, higher-order dispersion, and Kerr nonlinear coefficient of the chosen mode are calculated from the propagation constant and electromagnetic field distribution.

\subsection{Information Capacity Tool}

The information capacity of a communication channel is the highest rate at which information can be transmitted reliably through the channel. The well-known Shannon-Hartley theorem describes the maximum information rate of a linear 
channel with additive white Gaussian noise as a function of $(1 / 2) \log _{2}(1+\mathrm{S} / \mathrm{N})$, where $\mathrm{S} / \mathrm{N}$ is the signal-to-noise ratio of the communication signal to the noise and interference at the receiver.

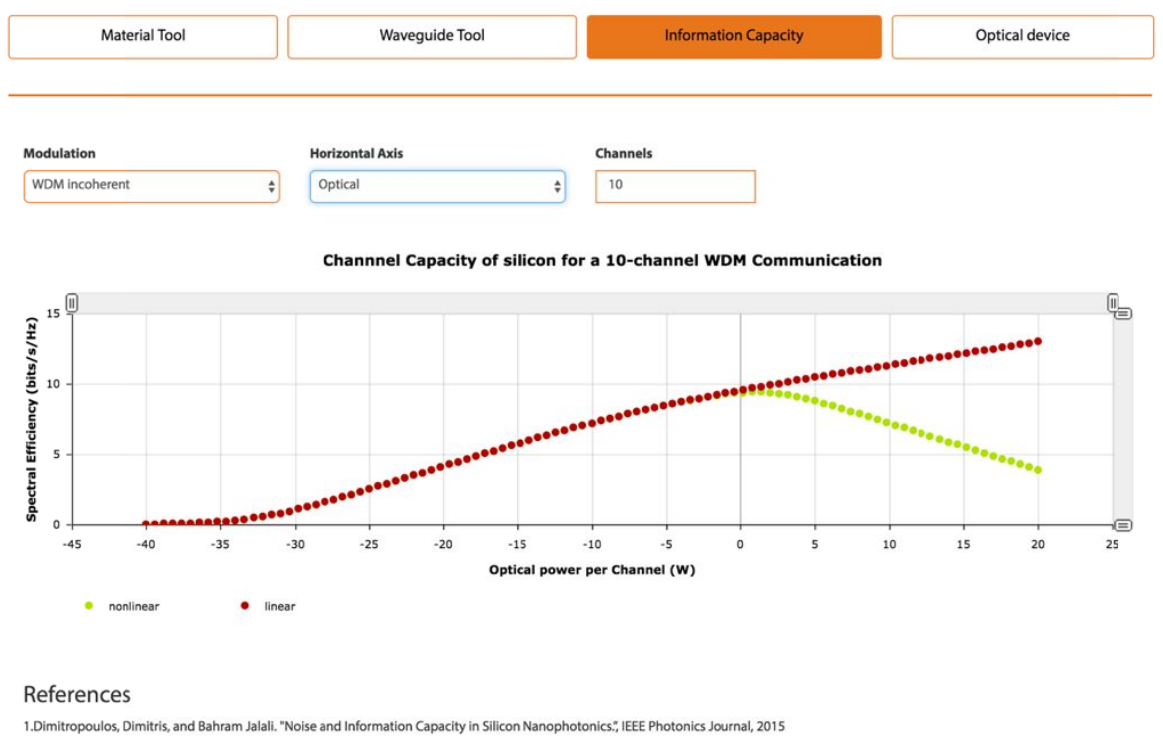

Figure 5. Information capacity of silicon waveguides in a 10-channel WDM communication characterized by linear model (red) and nonlinear model proposed in reference [11] (green). The latter shows the degradation of the capacity when crossphase-modulation takes place at high input power.

The information capacity calculation for silicon waveguides is however complicated by nonlinear losses, stemming from the strong power confinement in subwavelength scale. A model that characterizes the nonlinear noise and fluctuations in silicon waveguide, which was then utilized to estimate its information capacity was reported by Dimitropoulos and Jalali [11]. The information capacity tool provides interactive visualizations to enable the users to develop a quantitative and effective understanding of the fundamental theory.

\section{Conclusion ANd Future Scope}

Silicon photonics is an interdisciplinary field, encompassing material science, semiconductor physics, electronics, waveguide optics, and communication theory. It can be difficult for both students and researchers to get familiar with this interdisciplinary field and to visualize the impact of various different parameters on the performance of their designs. We demonstrated interactive online educational tools for the most fundamental linear and nonlinear properties of materials, waveguides, and information capacity in the field of silicon photonics.

We envision the Silicon Photonics Cloud to be a vertically integrated learning, and open-source platform, with modules spanning the full range from fundamental physics to abstract systems engineering. We foresee the future version of SiCloud to include basic passive and active optical devices. We are open to collaborations with researchers and educators in the field, to continue to provide effective and efficient tools in the various aspects of silicon photonics for the global audience.

\section{REFERENCES}

[1] Nikolova D., Rumley S., Calhoun D., Li Qi., Hendry R., Samadi P., and Bergman K., "Scaling silicon photonic switch fabrics for data center interconnection networks," Opt. Express 23, 1159-1175 (2015) 
[2] Wolfe J., "Why IBM and Intel are Chasing the \$100B Opportunity in Nanophotonics," Forbes, 2012 https://www.forbes.com/sites/joshwolfe/2012/12/13/why-ibm-and-intel-are-chasing-the-100b-opportunity-innanophotonics/\#24ee98fa4a69

[3] DeVore P.T., Jiang Y., Lynch M., Miyatake T., Carmona C., Chan A.C., Muniam K., Jalali B., "Silicon photonics cloud (SiCloud)". Proceedings of SPIE, vol. 9367, Silicon Photonics X, 93670G (2015).

[4] DeVore, P.T., Jiang, Y., Lynch, M., Miyatake, T., Carmona, C., Chan, A.C., Muniam, K., Adam, J. and Jalali, B., "Silicon photonics cloud (SiCloud)," 2015 2nd International Conference on Opto-Electronics and Applied Optics (IEM OPTRONIX), Vancouver, BC, 1-2 (2015).

[5] Palik, E.D., [Handbook of optical constants of solids], Vol. 3, Academic Press (1998).

[6] Reed, G. T., and Knights A.P., [Silicon Photonics: An Introduction], John Wiley \& Sons (2004).

[7] Jalali, B., "Silicon photonics: Nonlinear optics in the mid-infrared." Nature Photonics 4.8, 506 (2010).

[8] Hon, N.K., Soref R., and Jalali B., "The third-order nonlinear optical coefficients of Si, Ge, and Si1- x Ge x in the midwave and longwave infrared.", Journal of Applied Physics 110.1, 9 (2011).

[9] Tsang, H. K., and Y. Liu. "Nonlinear optical properties of silicon waveguides." Semiconductor Science and Technology 23.6, 064007 (2008).

[10] COMSOL Wave Optics Module Documentation, https://www.comsol.com/wave-optics-module - overview

[11]D. Dimitropoulos and B. Jalali, "Noise and Information Capacity in Silicon Nanophotonics," in IEEE Photonics Journal, vol. 7, no. 3, 1-20 (2015). 